\title{
Minimally invasive endoscopic transforaminal lumbar discectomy, a simplified technique guide
}

\begin{abstract}
Symptomatic degenerated spinal discs and spinal stenosis are common problems that can often be treated conservatively but some require decompressive spinal surgery for relief. Traditional open spinal discectomy is associated with significant tissue trauma, higher morbidity and complication rates, longer-term of convalescence, and even destabilization of the spine. The trend of spinal surgery is rapidly moving towards less traumatic minimally invasive spine surgery (MISS). ${ }^{1,2}$

The problem that faces the surgeon performing endoscopic MISS is that it is done with limited surgical exposure and visualization of the surgical field. The surgical field can only be viewed through an endoscope to correlate the lesion/pathology in relationship to imaging studies aided by C-arm fluoroscopy.

In response, a logical and simple Grid Positional System (GPS) was developed to provide a precise surgical trajectory/approach for the disc lesion to undergo decompression. GPS involves 3D geometric triangulation of 3 different planes guided by fluoroscopy for introduction of surgical instruments along a geometric line towards the lesion without compromising healthy anatomical structures. This system facilitates MISS, especially in the morbidly obese.

In this chapter, we will describe the GPS system and its application to aid in facilitating minimally invasive decompressive spine surgery for alleviating of symptoms of degenerative spinal disease, herniated disc, and spinal stenosis, while avoiding the complications and risks of conventional more traumatic spinal surgery and fusion.
\end{abstract}

\author{
Volume II Issue 2 - 2019
}

\author{
Ali M Maziad,' John C Chiu² \\ 'Consultant, Orthopedic Spine Surgery, California Spine \\ Institute, USA \\ ${ }^{2}$ Director, Neurospine Surgery, Department of Neurosurgery, \\ California Spine Institute, USA
}

\begin{abstract}
Correspondence: Ali M Maziad, Consultant, Orthopedic Spine Surgery, California Spine Institute, Thousand Oaks, California, USA, Email ali.maziad@gmail.com
\end{abstract}

Received: January 10, 2018 | Published: April 01, 2019

\section{Introduction}

Disc degeneration is a common problem affecting many middleaged individuals that increases in severity with age. Lack of exercise, bad postural habits, obesity and repetitive trauma may accelerate the process and can lead to early onset of symptoms. Depending on the number of spinal segments involved and the degree of degeneration, symptoms can range from mild muscular aches to severe neural compression that can compromise movement and sensation severely up to a point of disability. Treatment usually follows a hierarchy of steps or algorithm ranging from rest and medical treatment to extensive major surgery. Due to the nature of conventional spine surgery, large incisions and extensive deep tissue dissection are mandatory to gain access to the site of pathology.

Following open procedures, delayed recovery which maybe incomplete is expected. Extensive muscle dissection becomes a source of pain for those patients and recovery can take months before the patient adapts to the pain due to surgery and until the patient's spine achieves fusion. Quite often, posterior fusion alone is not sufficient to achieve a sound biomechanical fusion. Anterior inter-body fusion may be needed which in turn poses more surgical trauma and risks if done trans-abdominally. Patients are often reluctant to proceed with conventional extensive surgery due to its risks. Thanks to recent advances in imaging, endoscopic and laser systems, minimally invasive spinal surgery ${ }^{1-3}$ can be safely and effectively performed with far fewer risks. The goal of minimally invasive spinal surgery is to decompress nerve roots and disc bulges as well as provide more room for the thecal sac (in cases of spinal canal stenosis) through small access portals without destabilizing or interfering with the structure of the spine. In cases with gross instability of the spine, patients may still require spinal fixation. However, if the patient is high risk or refuses open surgery, minimally invasive spinal surgery with GPS should be the alternative surgical treatment.

We have applied the new concept of GPS (Grid Positioning System $)^{1}$ to precisely localize the lesion or target preoperatively and to aid in the localization of the foramen during surgery as well.

In this chapter we will demonstrate how GPS aids and facilitates ${ }^{1}$ minimally invasive spinal surgery ${ }^{4-12}$ in effectively alleviating the symptoms ${ }^{13-22}$ associated with degenerative disc disease and neurological compression with fewer risks and speedier recovery. ${ }^{23-33}$

\section{Surgical indications $s^{7-12}$}

a) Intractable low back pain with radiation down the leg (radicular pain).

b) Symptoms of spinal neurogenic claudication.

c) Compressive and symptomatic radiculopathy with sensory and motor impairment.

d) Disc extrusion or sequestration with predominantly back or leg pain.

e) Non-radicular low back pain persisting despite facet joint injection.

f) Lateral recess stenosis with dynamic compressive or noncompressive radiculopathy. 
g) Prior failed conventional surgery with perineural scarring and failed back syndrome and junctional disc degeneration and disc herniation following fusion.

h) No improvement of symptoms after a minimum of 12 weeks of conservative therapy.

i) Grade 1 spondylolisthesis.

j) Diffused multi-level disc degeneration that would otherwise require very extensive open surgery.

\section{Contraindications}

a) Cauda Equina syndrome.

b) Tumors.

c) Gross instability of the degenerative spine, (spondylolithesis Grade 2 and 3) - In those cases, minimally invasive spinal surgery (MISS) may not be the complete answer to the problem due to the biomechanical problem, however it may be the only alternative to an extensive surgery if the patient's general condition does not allow surgery.

d) Post traumatic unstable spinal fractures.

\section{Anesthesia and positioning ${ }^{7-12}$}

Unlike conventional surgery, minimally invasive spine surgery can be effectively done under local anesthesia and IV conscious sedation. We have modified our technique so that the patient is positioned in the lateral decubitus with the painful leg up and both hips and knees in 90 degrees of flexion for positioning and to reduce the traction on the nerve roots. Compared to prone position, the lateral position reduces intra-abdominal pressure, venous pressure, pelvic congestion and risk of DVT as well as pulmonary complications. The patient is positioned on one side first and then the other to perform a bilateral procedure in one sitting.

\section{Continuous intra-operative monitoring ${ }^{9-12}$}

Sterile needle electrodes are placed for continuous intraoperative neurophysiological electromyographic (EMG) monitoring. EMG monitoring is absolutely needed to avoid the risk of nerve root injury during MISS procedures. In addition EEG surface monitoring is utilized to optimize anesthesia and reduce anesthetic requirements. Full monitoring of the patient's vitals is also recommended even if procedure is done under local anesthesia.

\section{Localization by using GPS}

The Grid Positioning System (GPS) is an anatomical grid system utilized to accurately determine the exact position of the protruded or extruded disc fragment in a 24 zone grid. Understanding of the map of 24 zone of grid position in the lumbar vertebrae in relation to other anatomical landmarks such as the pedicle, lamina and thecal sac is critical and important to guide the surgical approach and to perform precise endoscopic MISS. Depending on the position of the protruded disc, different transforaminal trajectories (either ipsilateral or contralateral to the lesion) can be used to create a portal of entry and gain access to the pathology. Central lesions can also be accessed through interlaminar approach. Accurate preoperative localization of the lesion is important in order to precisely reach the lesion and avoid undue trauma to the nerves, vessels, dorsal root ganglia, dura and even the spinal cord (Figure 1).

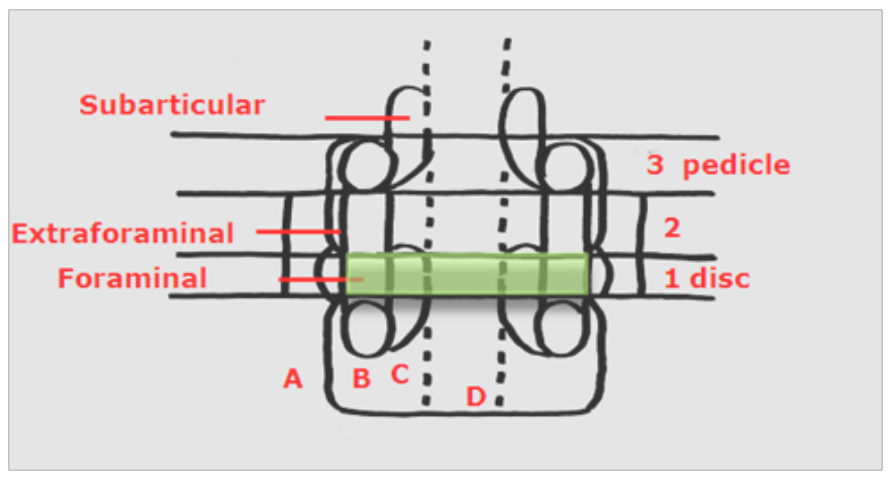

Figure I Grid Positional System (GPS) with various zones.

The location of the lesion or target can be precisely localized within the grid of the GPS System (Figure 1). Twenty four different zones $(\mathrm{A}, \mathrm{B}, \mathrm{C}, \mathrm{D}$ and $1,2,3)$ of the lumbar spine are located in the grid at these zones.

The four vertical zones of the lumbar vertebrae are:
A. ExtraForaminal
B. Foraminal
C. Subarticular
D. Para-central - (Right and left)

The three horizontal levels of the lumbar vertebrae are:

i. At the level of the disc.

ii. Between the disc and the pedicle, at the level of the neuroforamen.

iii. At the level of the pedicle.

\section{Surgical equipment, instruments (Figure 2) and preparation $^{7-11}$}

The following surgical instruments are necessary to perform endoscopic laser, and/or lumbar foraminoplasty, or both:

a. Digital fluoroscopic equipment (C-arm) and monitor.

b. Radiolucent C-arm/fluoroscopic carbon-fiber surgical table.

c. Endoscopic tower equipped with digital video monitor.

d. Wide-angle percutaneous posterolateral foraminoscope 6-degree, operating sheath 6-mm OD, 3-mm working channel.

e. Set of serial/progressive dilators, and cannula in graduated sizes $(3.5-5.8 \mathrm{~mm})$; a set of progressive cannula with duck bill extensions (with various lengths $5-10 \mathrm{~mm}$ on one side), to retract and to protect neural structures from neural trauma intraoperatively.

f. Aggressive toothed trephine set in graduated sizes.

g. 9.9-mm tubular retractor system with a gradual dilator set.

h. Wide angle endoscopes, 0-degree and 30-degree, 4-mm OD (Karl Storz).

i. Trephine, curette, grasper and spoon forceps, 2-mm rotating bone punch (rongeurs), rasp, and burr. 
j. Lumbar discectomy sets $(2.5,3.5,4.7 \mathrm{~mm})$.

k. Endoscopic grasping and cutting forceps, probe, knife, scissors, discectomy rongeurs, and curette (see Figure 1).
1. Holmium: yttrium aluminum garnet (YAG) laser generator.

m. Holmium 550- $\mu \mathrm{m}$ laser bare fiber with flat tip and right angle (side-firing) probes with and without irrigating system of various sizes, and 2-mm side-firing irrigating laser probe.
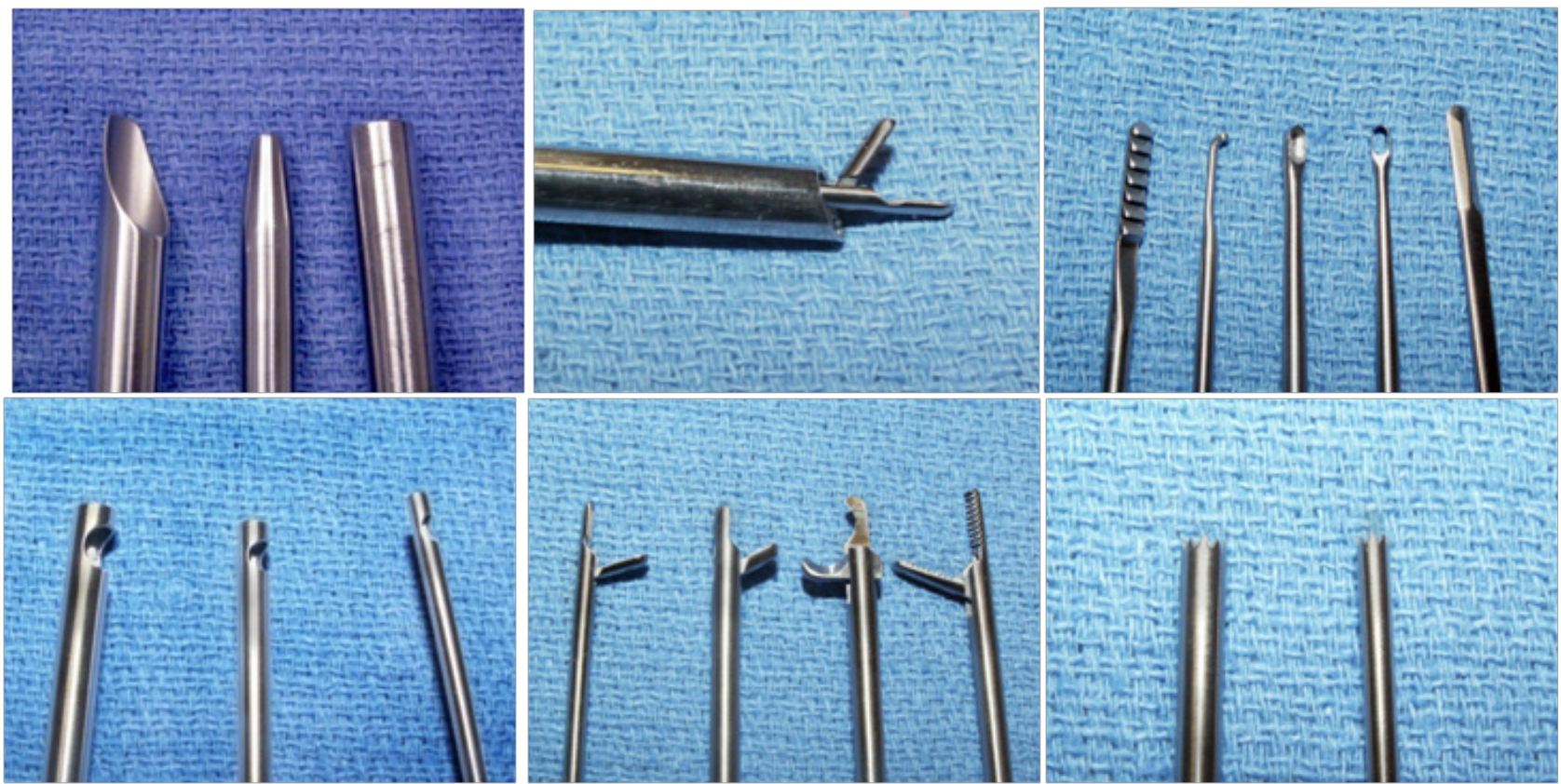

Figure 2 Different microdiscectomy instruments: duck bill tubular retractor with dilator, endoscopic grasper, rasps, curettes, discectomes, cutter and grasper forceps and trephine.

\section{Surgical procedure}

\section{Intra operative determination of portal of entry by GPS}

C-arm fluoroscopy is used to identify the lumbar levels requiring surgery. The midline of the spine (by connecting the lumbar spinal processes under fluoroscopy) and foraminal line of the spine (by connecting the neuro-foramen under fluoroscopy) are first identified by using a flexible stylette under fluoroscopy. A transverse disc space-line (representing the horizontal disc plane), is drawn under fluoroscopy, starting at the foramen line by using a flexible stylette and intersecting with the midline of the spine. The portal of entry for lumbar spinal surgery is marked on the transverse disc space-line with a marking pen. The distance of the point of the portal of entry from the midline varies with the height and weight of the patient. In an average-sized patient it is approximately $12 \mathrm{~cm}$ at the affected disc level. The more obese the patient, the greater the distance of the portal of entry point is from the midline in order to accommodate for excess subcutaneous fat.

\section{Operative technique 9,11}

Following GPS localization, local anesthetic is injected along the surgical trajectory the Kambin safety triangle to treat disc herniations (Figure 3-6, Figure 8, Figure 9). A long stylette is introduced through the identified entry point (as explained above) (Figure 10). The stylette is directed towards the disc through the Kambin Triangle (Safety zone) (Figure 3). The Kambin triangle is the site of surgical access for posterolateral endoscopic lumbar discectomy. It is defined as a right angled triangle over the dorsolateral disc. The hypotenuse is the exiting nerve, the base (width) is the superior border of the caudal vertebra, and the height is the traversing nerve root and dura.

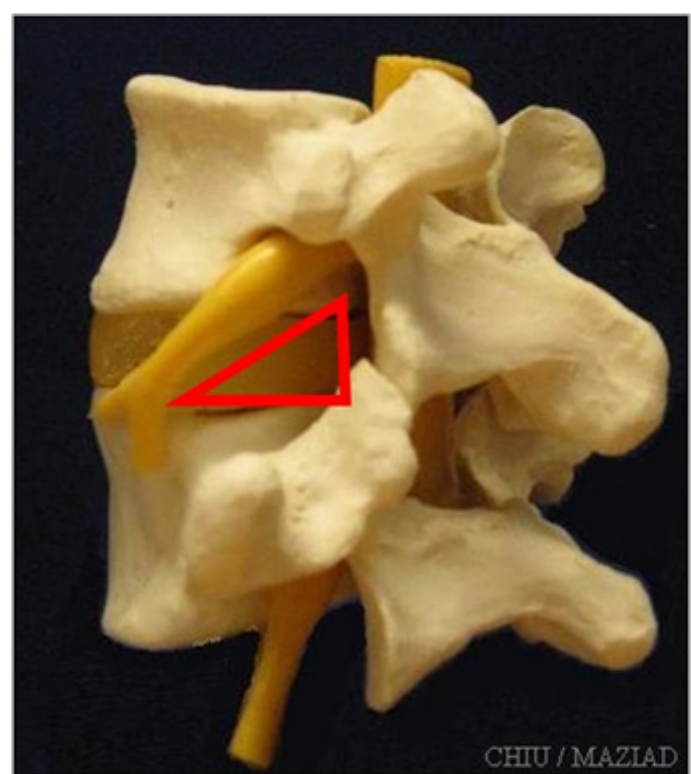

Figure 3 Kambin safety triangle.

a. Serial dilators are introduced guided by the stylette to reach the disc space (Figure 11a).

b. Provocative discogram is done to confirm disc pathology (Figure 11a,b).

c. Different types of graspers, trephines and curettes are utilized to mechanically decompress the disc material guided by fluoroscopy and endoscopy (Figure 11c-e,g, Figure 12, Figure $15 d)$. 
d. Bony spurs can also be trimmed using rongeurs, curettes or high power laser (Figure 13).

e. Following mechanical decompression, Holmium YAG laser is used for thermodiskoplasty of the disc which helps to Shrink the disc bulge, denervation of the pain fibers, seal the opening in the annulus fibrosis and coagulate bleeding vessels in vertebral endplates (Figure 13).

f. After laser application, discectome is used for suction irrigation of disc debris (Figure 14).

g. Steroid and anesthetic injection intradiscally and along the surgical tract is done as a final step to reduce postoperative pain and inflammation (Figure 15a).

h. A single stitch is used to close the skin puncture (Figure 15b,c).

i. At times, two levels can be accessed through a single skin puncture by changing the trajectory of the entry (Figure 8).

\section{Postoperative care $^{8-11}$}

Ambulation begins immediately after recovery, and the patient is usually discharged one hour after surgery. They may shower the following day. Applying an ice pack is helpful. Nonsteroidal antiinflammatory drugs (NSAIDs) are prescribed, and mild analgesics and muscle relaxants as needed. Patients return progressively to usual activities in ten days to three weeks, provided heavy labor and prolonged sitting are not involved.

\section{Case illustration}

A 63-year-old patient with morbid obesity and insulin dependent diabetes mellitus suffered from spinal canal stenosis and multiple level disc protrusions at L2/3, L3/4, L4/5 and L5/S1. The largest disc was at $\mathrm{L} 5 / \mathrm{S} 1,>6 \mathrm{~mm}$ as illustrated below. His weight is $280 \mathrm{lbs}$ and height 5' 8" (Figure 4-16). The patient complained of back pain and radicular symptoms including the anterior and surface of both thighs and legs, and back of the foot with more severe pain on the right side associated with some neurogenic claudication. Previously the patient refused open surgery with fusion, because of increased risk in morbid obese and diabetic patients and requiring a multi level extensive open spinal fusion. After reviewing his MRI scans, (Figure 4) minimally invasive endoscopic microdecompression surgery was recommended to the patient as a solution for his spinal condition. He agreed to proceed with MISS surgery. It can be very time consuming to reach the foramen in obese patients since the fat distribution gives a false orientation of the actual location of the spine in relation to the skin surface. By using the GPS system to triangulate the 3D orientation of the foramen under fluoroscopy, the height, width and depth of the soft tissue in relation to the foramen was determined the microdecompression was successfully performed. Bilateral L2L5 endoscopic microdecompressive discectomy (Figure 5-16) was performed in the lateral decubitus position on two sittings of less than 1 hour for each side to avoid a prolonged single procedure. The patient reported marked relief of his back symptoms and lower limb numbness on the next post operative day with a better range of motion and ambulation.

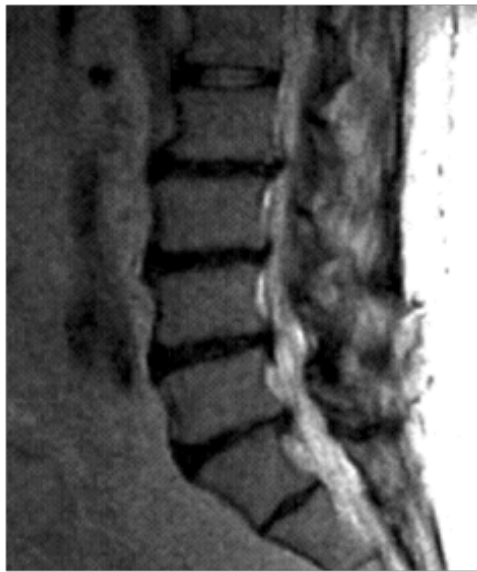

(a)

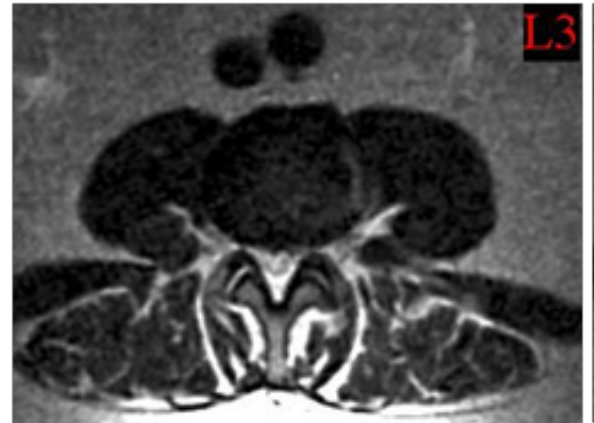

(d)

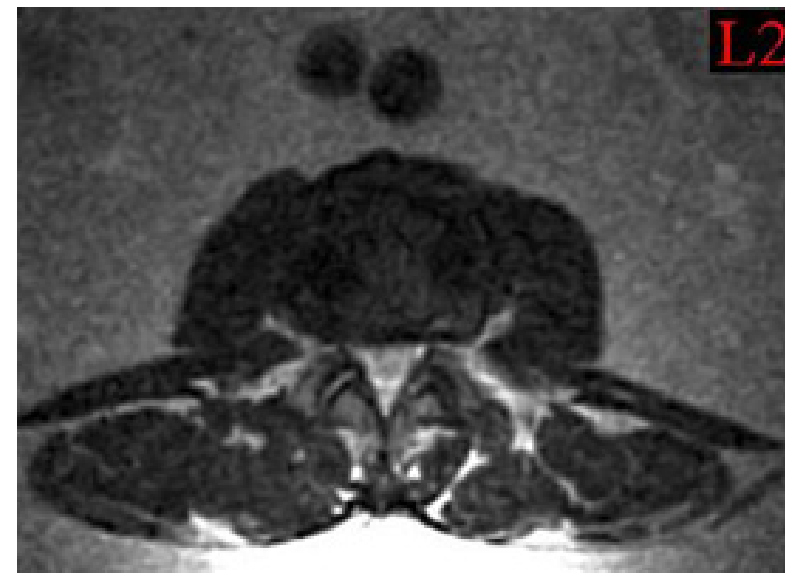

(b)

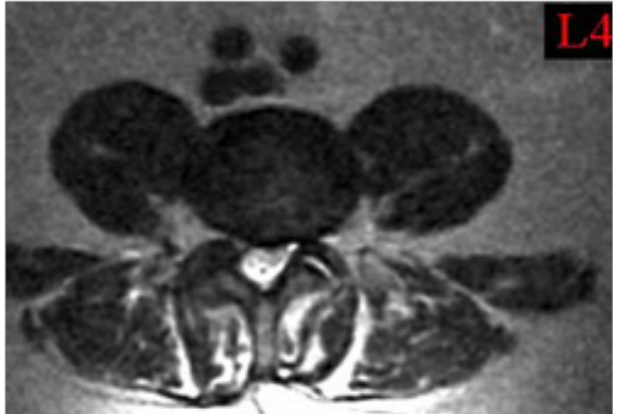

(e)

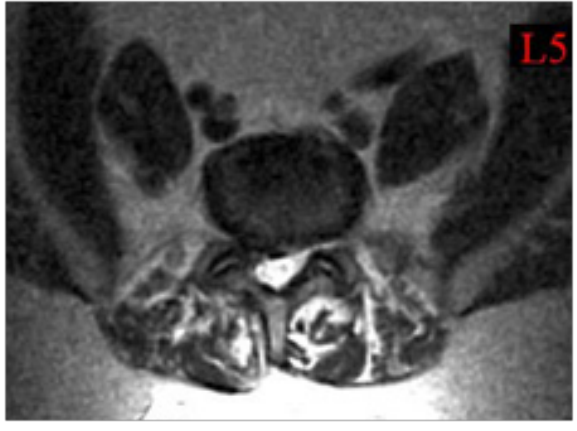

(f)

Figure 4 (a) Sagital T2 view MRI showing multi-level disc protrusions and canal stenosis (b, c, d, e) with bilateral nerve roots compression. 


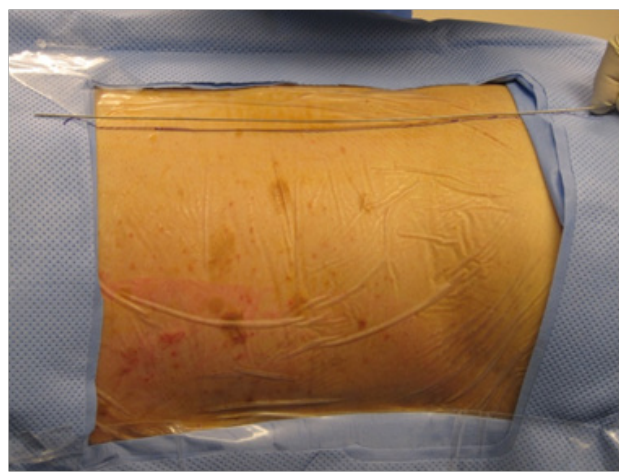

(a)

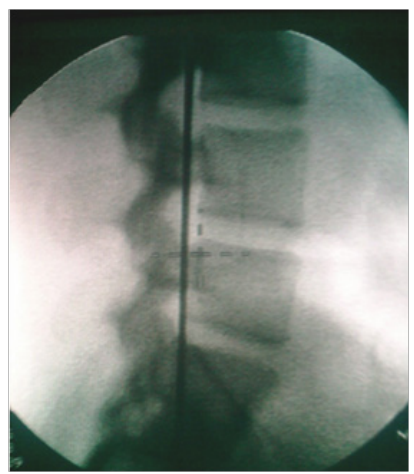

(b)

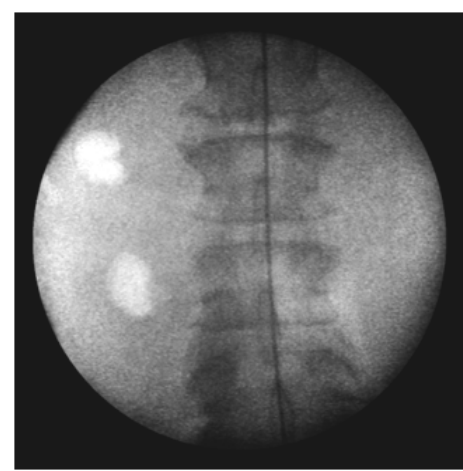

(c)

Figure 5 (a) Identifying the foraminal line on patient (b) Fluoroscopic lateral view (c) Fluoroscopic midline or lateral foraminal line (On APView).

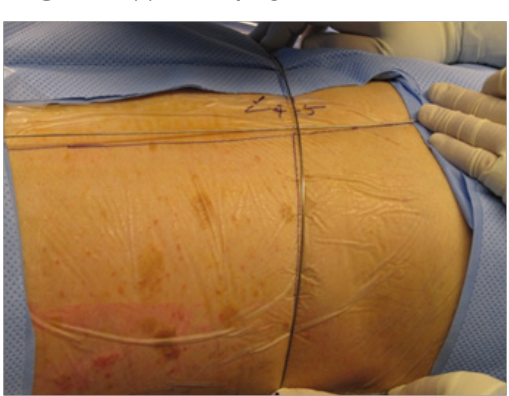

(a)

Figure $6(\mathrm{a}, \mathrm{b})$ Flexible stylette is used to identify the intersection of the L4/5 disc space line with the foraminal line, on patient and under fluoroscopy.

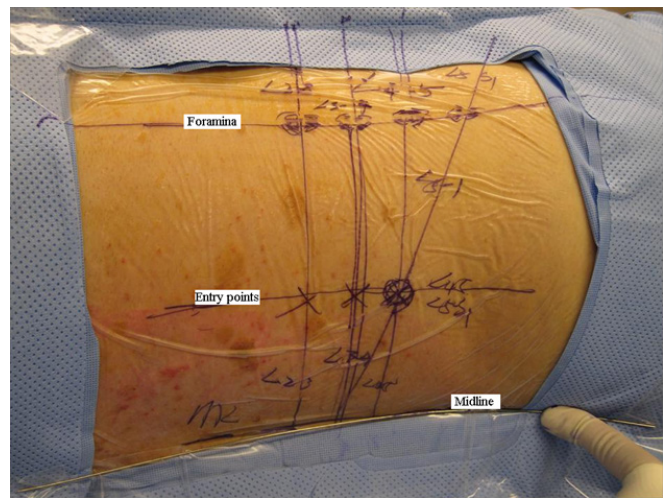

Figure 8 Completed GPS (grid positional system).

Note: L4/5 disc portal of entry point can be used to access both L4/5 and L5/SI discs by applying different angles to the trajectory of the stylette, perpendicular (for L4/5 disc) vs. steeper angle (for L5/SI disc).

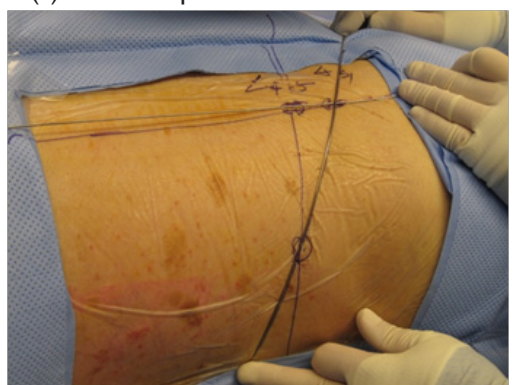

(a)

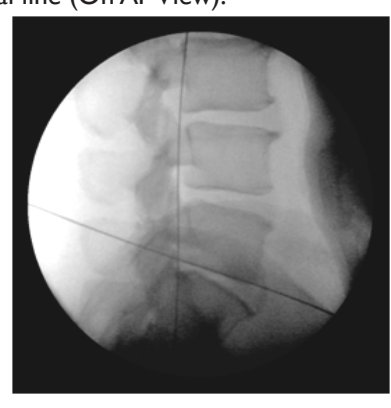

(b)

Figure 7 (a, b) L5/SI disc can be accessed through the same skin puncture entry through a steeper angle, on patient and under fluoroscopy.

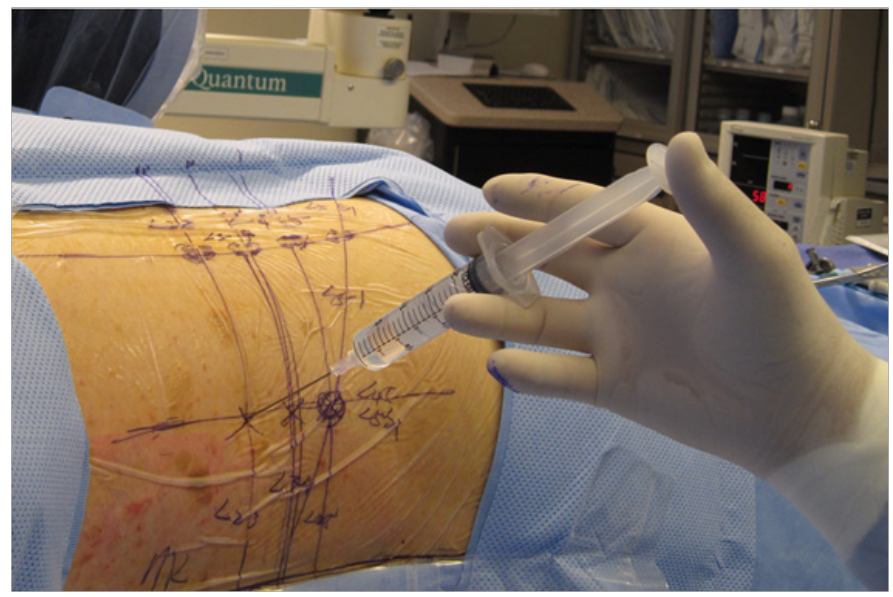

Figure 9 Local anesthetics is injected for each portal entry point.

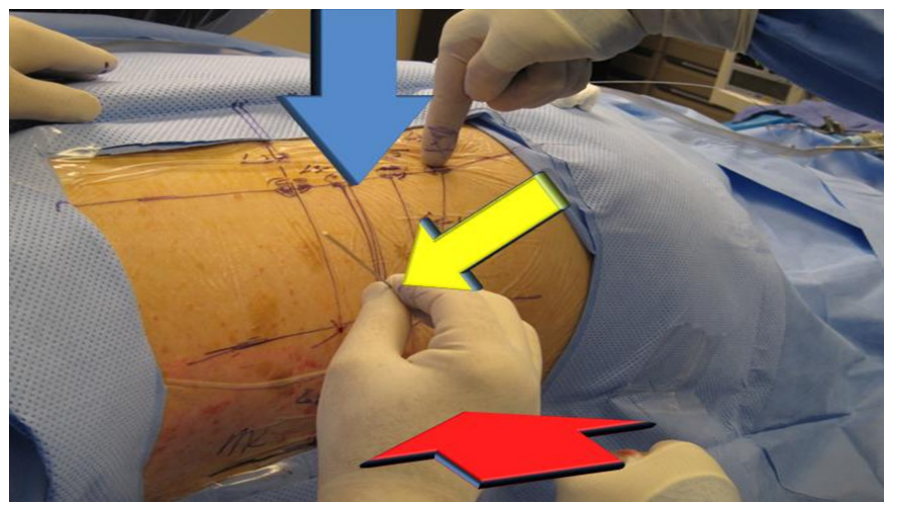

Figure 10 Stylette is introduced at an angle of approximately 45 degrees accurately toward the foramen in the GPS grid, under fluoroscopy.

Citation: Maziad AM, Chiu JC. Minimally invasive endoscopic transforaminal lumbar discectomy, a simplified technique guide. MOJ Orthop Rheumatol. 2019; II(2):91-99. DOI: 10.15406/mojor.2019.II.00479 


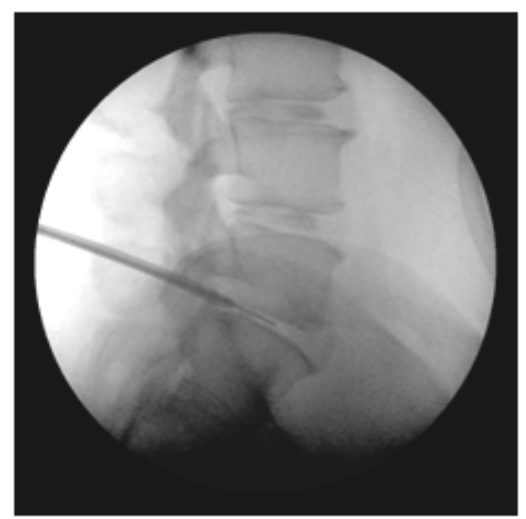

(a)

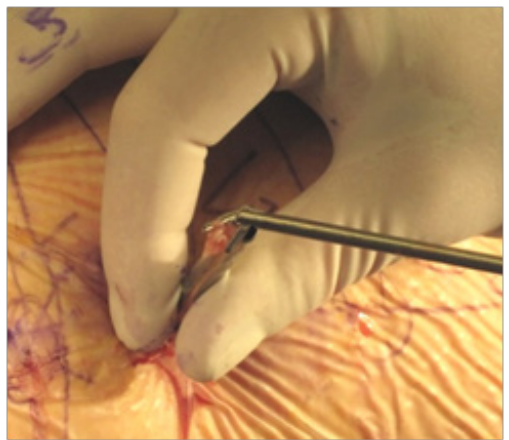

(d)

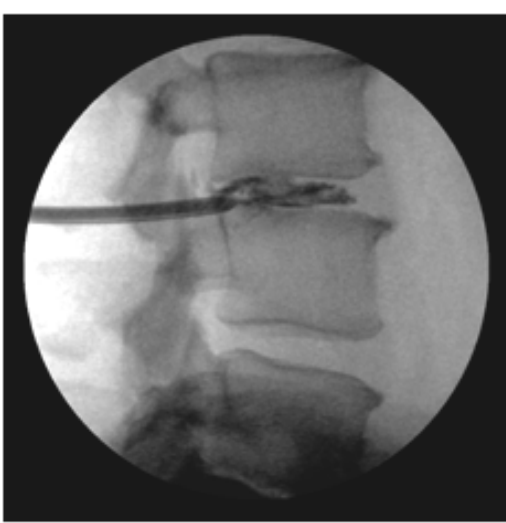

(b)

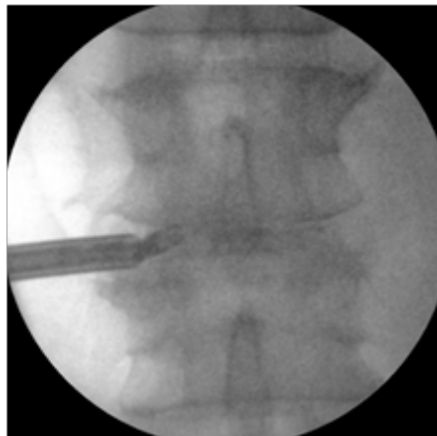

(e)

(f)

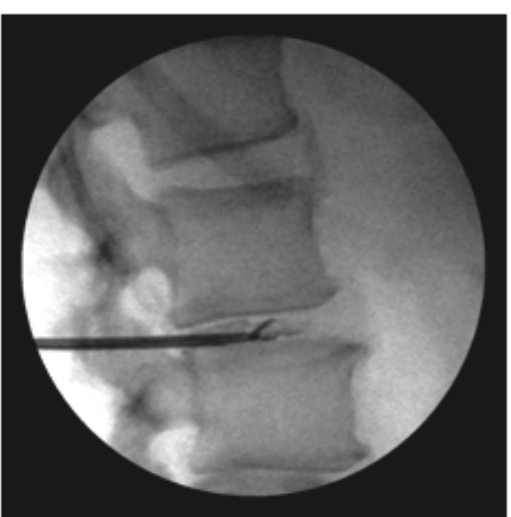

(c)

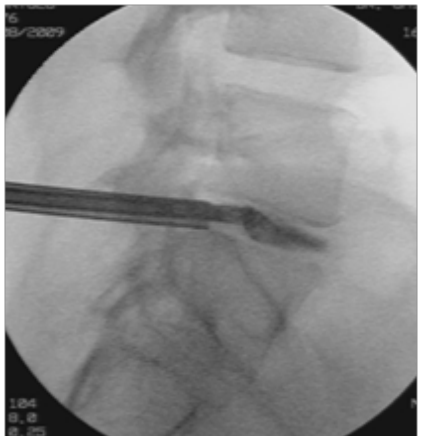

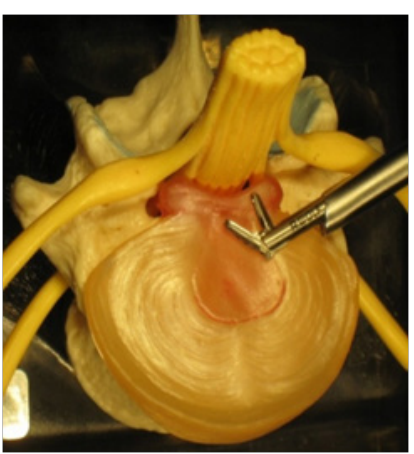

(g)

Figure I I Microdecompressive lumbar discectomy fluoroscopic views of a. stylette and dilator, b. Discogram confirming disc degeneration, c. Grasping forceps, d. ,e., f., g. Using a special flexible articulating angle grasper to reach posterior protruded herniated disc not reached by a conventional grasper.
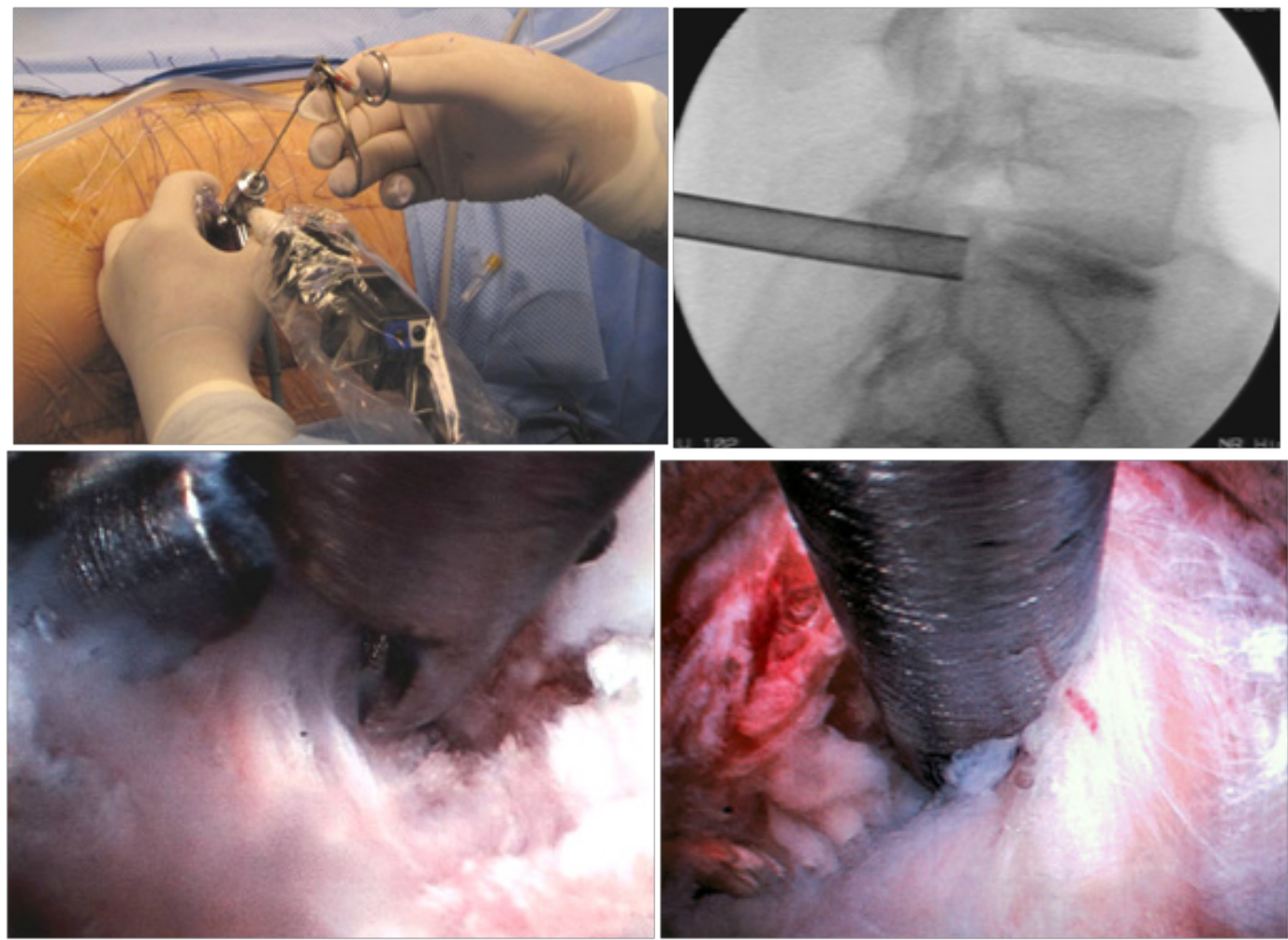

Figure I 2 Performing endoscopic disc micro decompression.

Citation: Maziad AM, Chiu JC. Minimally invasive endoscopic transforaminal lumbar discectomy, a simplified technique guide. MOJ Orthop Rheumatol. 20 I9; I (2):9|-99. DOI: I0.15406/mojor.20 I9.I I.00479 


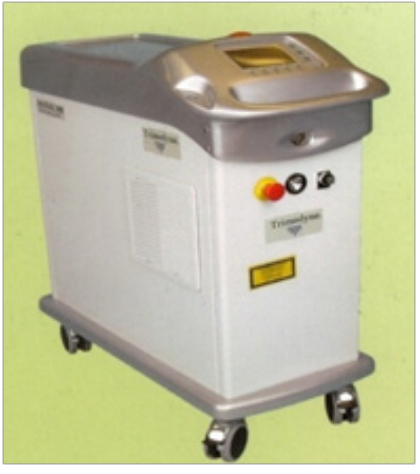

(a)

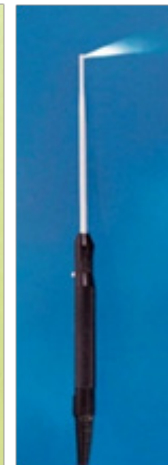

(b)

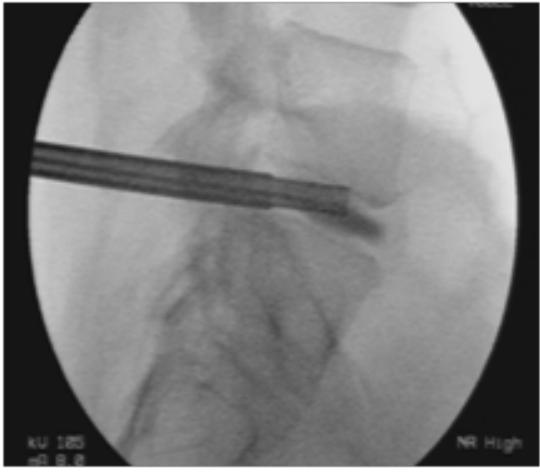

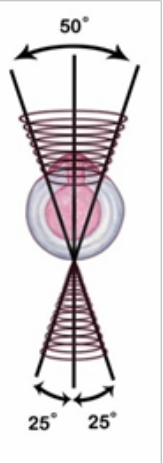

(c)

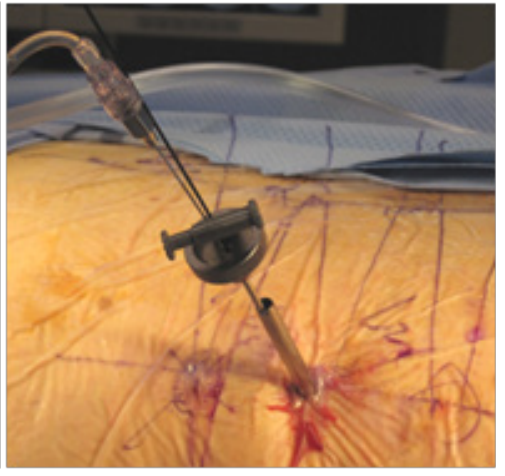

(d)

Figure I3 a, b, c, d Holmium YAG laser generator, probe, on fluoroscopic view, inverted cone shape maneuver, laser thermodiskoplasty procedure.
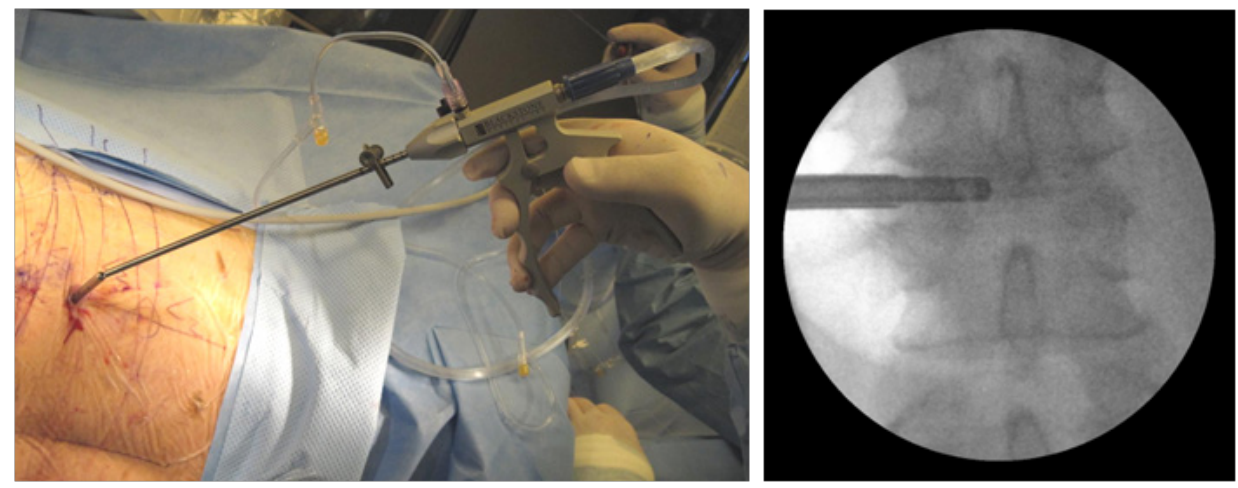

Figure I4 Discectome for irrigation and suction of debris after laser application and fluoroscopic view.
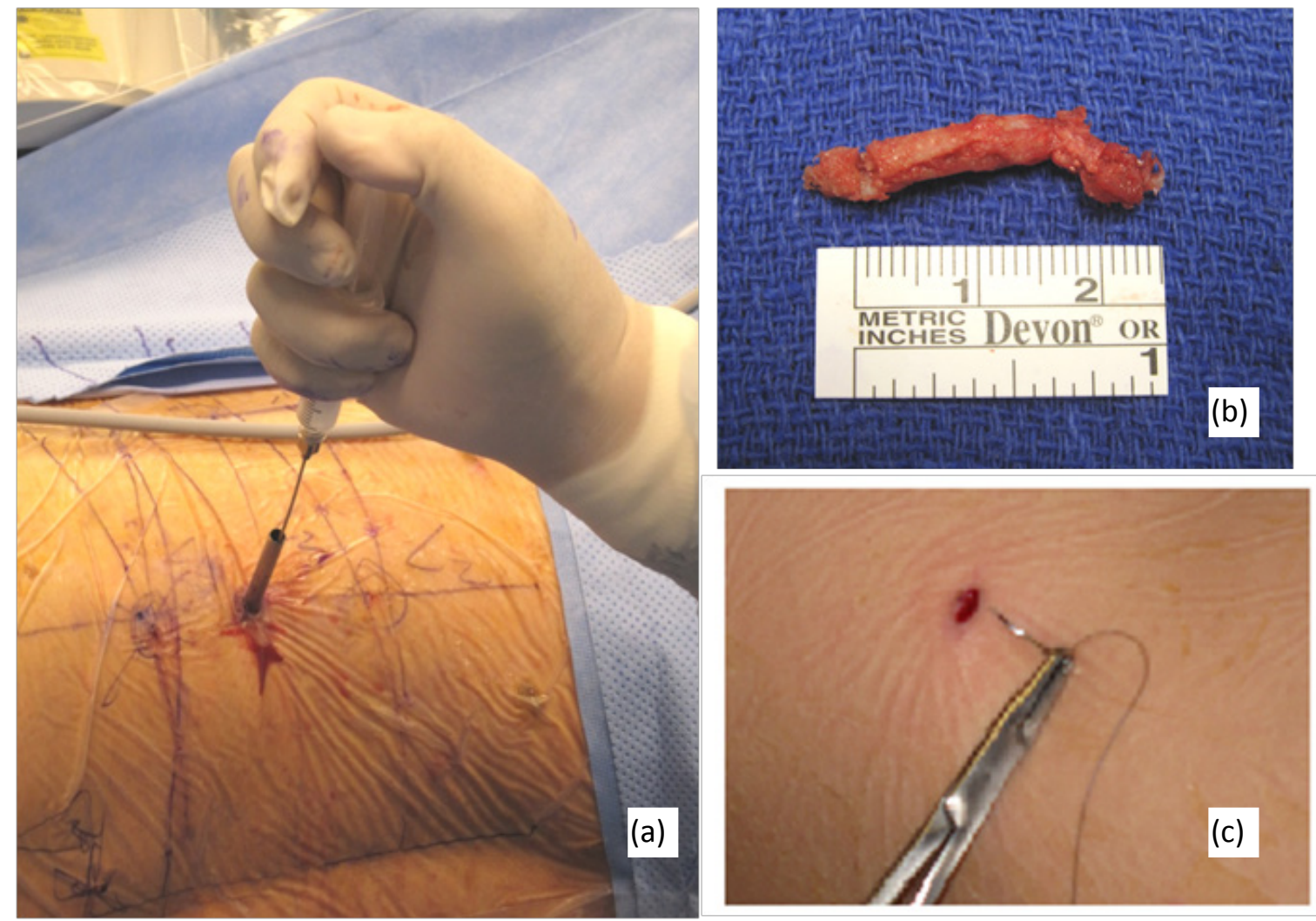

Figure $\mathbf{I 5}$ a. Steroid and anesthetic injection into the disc as final step to reduce postoperative pain and inflammation b. Excised disc material and calcified osteophytes of the affected level c. Single stitch to close the skin puncture wound. 

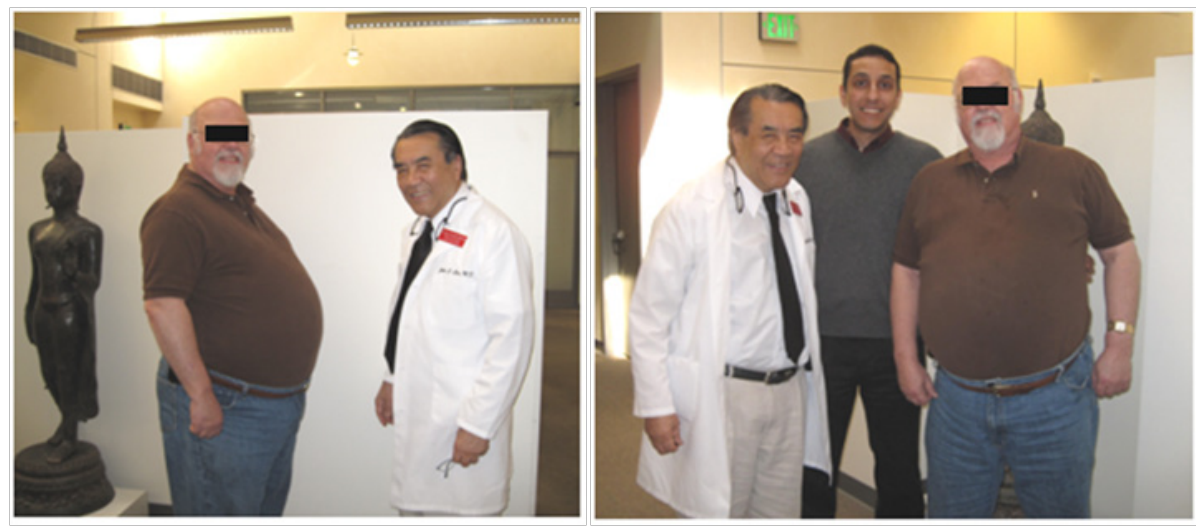

Figure 16 Morbidly obese patient immediately following successful multi-level GPS microdecompressive lumbar discectomy.

\section{Discussion}

We have developed a step by step localization system (Grid Positioning System or GPS) to facilitate the precise surgical approach toward the lesion or target for MISS procedure. Utilizing GPS dramatically improves accuracy, reduces the operative time and adds an extra advantage on an improved surgical outcome particularly in high risk medical patients. Minimally invasive endoscopic microdecompression for herniated lumbar discs and spinal stenosis can effectively treat disc pathologies and spinal stenosis and alleviate patient symptoms. However, accurately localizing the spinal foramen and lesion can be a challenging tedious task for the inexperienced surgeon particularly in cases with abnormal anatomy following previous open surgery or spinal deformities such as degenerative scoliosis. More commonly localizing the spinal foramen can be difficult in morbidly obese patients where the topographic anatomy related to the spine is quite different from the norm. Again, GPS facilitates the MISS process.

MISS with GPS guidance combines different modalities for addressing spinal pathology; aggressive mechanical decompression, laser application, disc suctioning and pharmacological treatment of the disc by steroids and anesthetics to reduce inflammation. It can effectively treat spinal pathology at multiple levels without having to worry about the potential complications of extensive exposures, prolonged surgery, infection, implant failure, potential junctional disc herniation often associated with conventional spinal surgeries. It also provides significant economic savings as no hardware implants are required, recovery is much faster, easier and fewer medications are needed.

Furthermore, this is the only logical solution for many elderly patients (even octogenarians and beyond) who suffer symptoms caused by spinal stenosis and disc problems in order to avoid major surgery. The results of this operation can be an extremely gratifying experience for both the patient and the surgeon.

Although MISS with GPS guidance is not the answer to all spinal problems, we believe it can address many of the problems related to spine degeneration without destabilizing the spine or compromising the flexibility of the motion segment if clinically appropriate, we recommend treatment with MISS followed by several months of recovery and re-evaluation before a patient under goes an irreversible fusion surgery.

The MISS procedure with GPS guidance requires a knowledgeable and competent surgeon with a thorough understanding of the surgical anatomy of the spine. Therefore, we believe that previous surgical experience with conventional open procedures can actually help the surgeon better understand this type of surgery compared to surgeons without such experience. A minimally invasive spine surgeon must have surgical training with hands-on experience with an expert in the field in order to go through the steep surgical learning curve.

Minimally invasive spinal surgery with GPS guidance is the logical evolution of spine procedures. With the current fast paced advancement in surgical technology, more applications for MISS will emerge in the near future. ${ }^{1}$

\section{Conclusion}

Applying the concept of Grid Positional System (GPS) to minimally invasive spinal surgery procedures can help the surgeon to facilitate the MISS process by quickly identifying the surgical portal of entry to the disc or target without compromising vital anatomical or neural structures and accomplish needed spinal microdecompression. GPS is of particular value especially in medically high risk patients including the morbidly obese and even those with prior surgeries. With the aid of GPS, MISS procedures can be very effective in surgical treatment of degenerative spine; especially if proper patient selection and sound MISS techniques are utilized.

\section{References}

1. Chiu JC, Maziad AM. Innovative Grid Positional System (GPS) Guidance for Minimally Invasive Spinal Surgery. Surg Technol Int. 2010;20(1):363-371.

2. Chiu J, Maziad A, Rappard G, et al. Evolving Minimally Invasive Spine Surgery: a Surgeon's Perspective on Technological Convergence and Digital OR Control System. In: Szabo Z, et al. editors, Surgical Technology International XIX, UMP, San Francisco, CA; 2009. p.211222.

3. Chiu J. Surgeon's Perspective and Consideration: OR Digital Technology Convergence and Control System for Minimally Invasive Spine Surgery, Presented at Special Session, Minimally Invasive Spine Surgery, CARS 2008, Computer Assisted Radiology and Surgery 22nd International Congress and Exhibition, Barcelona, Spain, Proceedings;2008. 23-28.

4. JC Chiu. A surgeon's perspective on digital technological convergence and control system for minimally invasive spine surgery, abstract published in Computer Assisted Radiology and Surgery, Proceedings of the 24th International Congress and Exhibition, Geneva, Switzerland, Springer Verlag, Heidlberg;2010.5(1):S5-S10.

5. Chiu J. Digital Technology Convergence and Control System: Minimally Invasive Spine Surgeon's (MISS) Perspective and Technological Consideration, "Interdisciplinary PACS" The Second Iranian Imaging Informatics Conference Syllabus, Tehran, Iran, 2008. pp 30-31. 
6. Chiu J, Therapeutic Application of Surgical ePR Control System beyond Radiology PACS, presented at the SPIE Medical Imaging Advanced PACS Based Imaging Informatics and Therapeutic Applications, Orlando, Fl, 2009. 8-12.

7. Chiu J, Savitz MH. Use of Laser in Minimally Invasive Spinal Surgery and Pain Management. In: Kambin P, ed. Arthroscopic and Endoscopic Spinal Surgery - Text and Atlas. Second Edition. New Jersey: Humana Press; 2005: Chapter 13, pp 259-269.

8. Chiu J. Anterior Endoscopic Cervical Microdiscectomy. In: Kim D, Fessler R, Regan J, editors. Endoscopic Spine Surgery and Instrumentation. New York: Thieme Medical Publisher;2004: Chapter 5, pp 48-58.

9. Chiu J. Posterolateral Endoscopic Thoracic Discectomy. In: Kim D, Fessler R, Regan J, editors. Endoscopic Spine Surgery and Instrumentation. New York: Thieme Medical Publisher; 2004: Chapter 11, pp 125-136.

10. Chiu J, Clifford T, Princenthal R. Junctional Disc Herniation in Post Spinal Fusion Treated with Endoscopic Spine Surgery: In, Szabo Z, Coburg AJ, Savalgi R, Reich H, Yamamotto M, editors. Surgical Technology International XIV, UMP, San Francisco, CA 2005: p.305315 .

11. Chiu J. Endoscopic Assisted Microdecompression of Cervical Disc and Foramen In, Szabo Z, Coburg AJ, Savalgi R, Reich H, Yamamotto M, editors. Surgical Technology International XVII, UMP, San Francisco, CA 2008: p.269-279.

12. Chiu J. Endoscopic Assisted Lumbar Microdecompressive Spinal Surgery with a New Smart Endoscopic System. In, Szabo Z, Coburg AJ, Savalgi R, Reich H, Yamamotto M, editors. Surgical Technology International XV, UMP, San Francisco, CA 2006 : p. 265-275.

13. Chiu, J. Cervical Endoscopic Microdecompressive Discectomy and Foraminal Decompression, Presented at the annual meeting of the Rome Spine Society, Rome, Italy, 2009 006: p.265-275.

14. Savitz MH, Chiu JC, Yeung AT. History of Minimalism in spinal medicine and surgery. In: Savitz MH, Chiu JC, Yeung AD (eds), The practice of minimally invasive spinal technique. Richmond, VA: AAMISMS Education, LLC; 2000. pp 1-12.

15. Chiu JC. The Decade of Evolving Minimally Invasive Spinal Surgery (MISS) and Technological Considerations. The Internet Journal of Minimally Invasive Spinal Technology. 2008.

16. Liu BJ, Law YY, Documet J, et al. Image-Assisted Knowledge Discovery and Decision Support in Radiation Therapy Planning, Computerized Medical Imaging and Graphics, 2007. 31:4-5, pp. 311-321.

17. Hijikata S. Percutaneous nucleotomy: A new concept technique and 12 years' experience. Clin Orthop Relat Res. 1989;238:9-23.

18. Ascher PW, Choy D. Application of the laser in neurosurgery. Laser Surg Med. 1986;2:91-97.

19. Kambin P, Saliffer PL. Percutaneous lumbar discectomy: reviewing 100 patients and current practice Clin Orthop Relat Res. 1989;238:24-34.

20. Schreiber A, Suezawa Y, Leu HJ. Does percutaneous nucleotomy with discoscopy replaces conventional discectomy? Eight years of experience and results in treatment of herniated lumbar disc. Clin Orthop Relat Res. 1989;238:35-42.
21. Chiu J. Endoscopic Assisted Lumbar Microdecompressive Spinal Surgery with a New Smart Endoscopic System. In, Szabo Z, Coburg AJ, Savalgi R, Reich H, Yamamotto M, editors. Surgical Technology International XV, UMP, San Francisco, CA 2006: p.265-275.

22. Destandau J. Endoscopically assisted microdiscectomy. In: Savitz MH, Chiu JC, Yeung AD (eds), The practice of minimally invasive spinal technique. Richmond, VA: AAMISMS Education, LLC; 2000. pp 187192.

23. Chiu J. Evolving Transforaminal Endoscopic Microdecompression for Herniated Lumbar Discs and Spinal Stenosis: In, Szabo Z, Coburg AJ, Savalgi R, Reich H, eds. Surgical Technology International XIII, UMP, San Francisco, CA; 2004: pp. 276-286

24. Chiu J. Endoscopic Lumbar Foraminoplasty In: Kim D, Fessler R, Regan J, eds. Endoscopic Spine Surgery and Instrumentation. New York: Thieme Medical Publisher; 2004: Chapter 19, pp 212-229.

25. Chiu J, Clifford T, Princenthal R. The new frontier of minimally invasive spine surgery through computer assisted technology. In: Lemke HU, Vannier MN, Invamura RD, editors. Computer assisted radiology and surgery, CARS 2002. Berlin: Springer-Verlag; 2002. pp 233-237.

26. Chiu J, Clifford T. Microdecompressive percutaneous discectomy: Spinal discectomy with new laser thermodiskoplasty for non extruded herniated nucleus pulposus. Surg Technol Int. 1999;8:343-351.

27. Chiu J, Stechison M. Percutaneous Vertebral Augmentation and Reconstruction with an Intervertebral Mesh and Morecelized Bone Graft: In, Szabo Z, Coburg AJ, Savalgi R, Reich H, Yamamotto M, editors. Surgical Technology International XIV, UMP, San Francisco, CA 2005: p.287-296.

28. Chiu JC, Hansraj K, Akiyama C, et al. Percutaneous (endoscopic) decompressive discectomy for non-extruded cervical herniated nucleus pulposus. Surg Technol Int. 1997;6:405-411.

29. Kambin P, Casey K, O’Brien E, et al. Transforaminal arthroscopic decompression of lateral recess stenosis. J Neurosurg. 1996;84:462-467.

30. Chiu JC, Clifford T. Multiple herniated discs at single and multiple spinal segments treated with endoscopic microdecompressive surgery. J Minim Invasive Spinal Tech. 2001;1:15-19.

31. Knight M, Goswami A. Endoscopic laser foraminoplasty. In: Savitz MH, Chiu JC, Yeung AD (eds), The practice of minimally invasive spinal technique. Richmond, VA: AAMISMS Education, LLC; 200. pp 337340 .

32. Clifford T, Chiu JC, Rogers G. Neurophysiological monitoring of peripheral nerve function during endoscopic laser discectomy, J Minim Invasive Spinal Tech. 2001;1:54-57.

33. Ciu J. SMART Endolumbar System for Microdecompression of Degenerative Disc Disease, presented at the Practical Course on Minimally Invasive Technique in Spinal Surgery, Russian Spinal Cord Society, Moscow Russia - April 26-29, 2007.

34. Chiu J. Complications and Avoidance in Endoscopic Spine Surgery, presented at the North American Spine Society Minimally Invasive Spine Technique: Hands-on Course, Barrow Neurological Institute (BNI), Phoenix, AZ. 